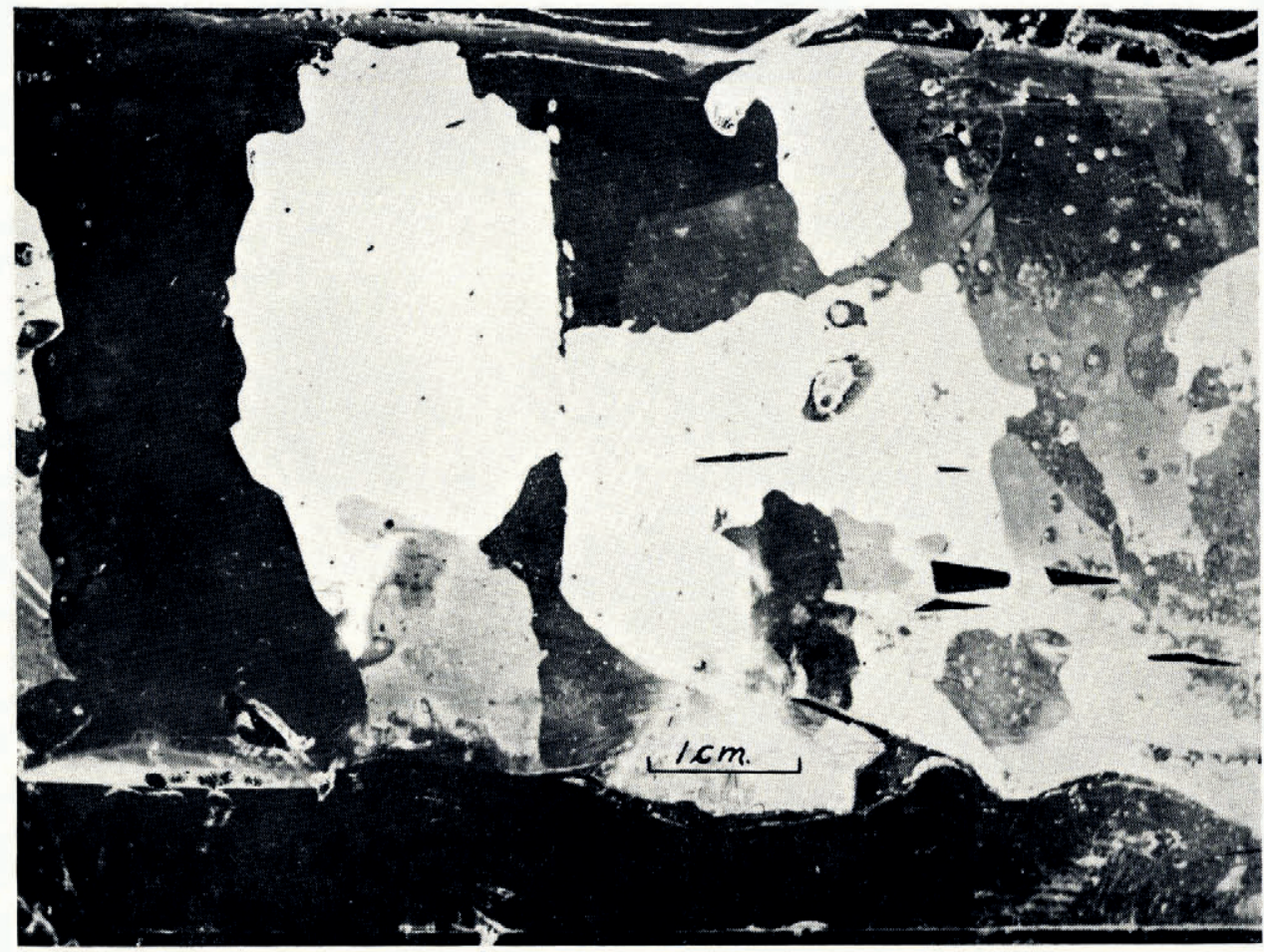

Fig. I

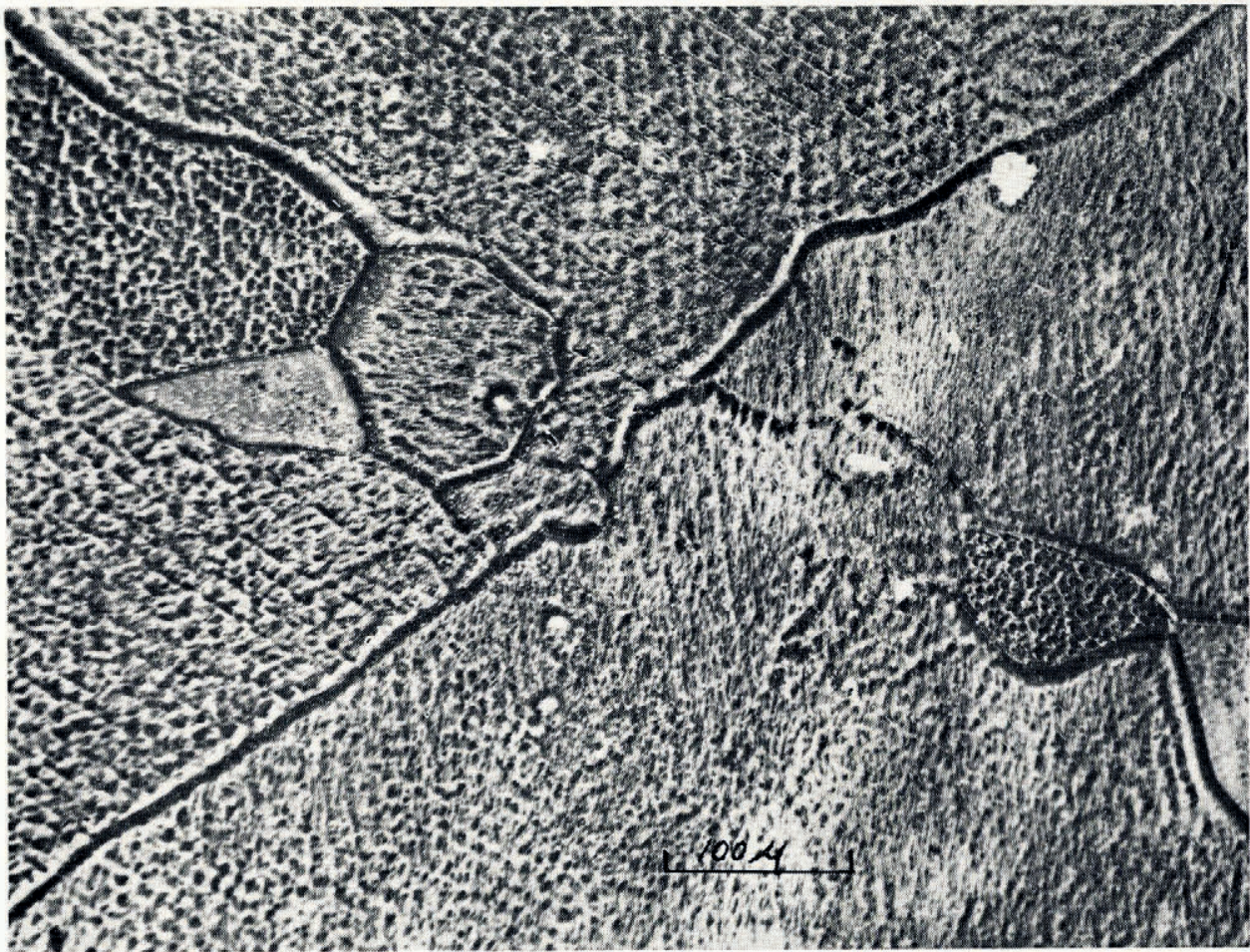

Fig. 2

Formvar replicas of glacier ice; see text opposite 


\title{
A NEW METHOD FOR STUDYING THE STRUCTURE OF GLACIER ICE
}

\author{
By Vincent J. Schaefer \\ (General Electric Research Laboratory, Schenectady, New York)
}

THE structure and motion of glaciers has been a subject of interest for many years. Although much scientific study has been directed towards this important natural phenomenon, many problems remain unsolved.

The change in structure which an air-borne ice crystal must undergo to become a part of the flowing greenish-blue mass of glacier ice involves processes which may require many years for final development. To understand the various factors involved in this transformation, it is desirable to employ new methods and techniques when they become available.

A considerable improvement for the study of the grain structure in glacial ice was described by Ahlmann and Droessler using a clay and absorbent paper technique. ${ }^{1}$ This was an improvement over the paper and pencil rubbing method of Seligman. ${ }^{2}$

During a visit to the Snow and Avalanche Research Institute of Switzerland * located on the Weissfluhjoch above Davos Dorf, the writer had an opportunity to study samples of glacial ice secured by Dr. Marcel de Quervain, Director of the Institute. These ice samples were taken from the walls of an artificial tunnel cut into the interior of the Z'mutt Glacier near Zermatt, Switzerland. In this brief study, an effort was made to apply the formvar replica technique developed by the writer for studying metallographic specimens in light and electron microscopes. ${ }^{3,4,5}$

A very simple process was developed permitting the preparation of a permanent and detailed record of the grain structure of glacial ice by the replica process. Such replicas not only show the grain boundaries but also much fine structure within the individual grains. Fig. I (p. 440) is a macrophotograph of a formvar replica of a small slab of glacial ice and shows the refraction and scattering produced when the replica was illuminated with a beam of parallel light. Fig. 2 is a photomicrograph of a similar replica illustrating the internal structure within a few of the smaller grains. The preparation of the surface of the ice samples followed typical metallographic techniques such as are used in preparing metals for micrographic study. A face of the ice was reduced to a plane surface by sawing and then grinding with successively finer grades of sharp abrasive on paper. This was followed by polishing on velvet, chamois and paper. In all cutting and polishing operations care was exercised in preventing excessive heating. With ordinary care this may be accomplished at a temperature of $-10^{\circ} \mathrm{C}$. or colder.

Etching of the surface was achieved by merely holding the sample below freezing with the air unsaturated with respect to ice. Slow evaporation of the surface molecules of ice over a period of thirty minutes at a temperature of $-10^{\circ} \mathrm{C}$. produced the "etching" illustrated in the figures.

The replica was made by coating the etched surface with a 3 per cent solution of polyvinyl formal dissolved in ethylene dichloride. At $-10^{\circ} \mathrm{C}$. the solvent evaporated in five minutes. As soon as the solvent had evaporated, the edge of the replica was "teased" loose with sharp-edged forceps, the edge lifted, and the replica sheet stripped off. (With time, the replica will become loosened automatically as evaporation proceeds through the film.) The stripped replica was then laid with its outer, smooth surface in contact with a glass sheet, a few drops of water placed at the interface, and the film spread out to its former area. Mounting is still easier if the film is floated on a cleaned water surface with the replica surface upward and then mounted by raising the glass plate into contact with it from the underside. A trace of gelatine or similar adhesive in the water will prevent subsequent peeling as the film dries. 
Various procedures may be used in employing replicas for studying ice samples. One of the most informative is to prepare a series of replicas representing planes through the ice at two or three millimeter intervals. This is accomplished by removing two or three millimeters of ice between each polishing operation. Such replicas piled on top of one another disclose the three-dimensional structure of the ice grains, and studies of this sort may provide valuable information relative to the forces and mechanisms leading to the formation and transformation of ice grains in a massive structure. Use of replicas containing dye ${ }^{6}$ should be particularly useful in some of this research.

The study of glacier ice, in addition to throwing more light on the problems concerning the flow of glaciers, might lead to some fundamental discoveries in the field of crystal structure and metallography. It is such a simple system; the method for study is straightforward; the polishing process is very easy, and the etching technique is free of mechanical distortion. Control observations may be maintained due to the transparent nature of the substance and its birefringent nature.

The writer is greatly indebted to Dr. de Quervain for his kindness in allowing him the freedom of his laboratory on the Weissfluhjoch and permission to try out the replica techniques on his polished samples of glacier ice from the Z'mutt Glacier.

\section{R E F E R E N C E S}

I. Ahlmann, H. W. and Droessler, E. G. Glacier Ice Crystal Measurements at Kebnekajse, Sweden. Fournal of Glaciology,

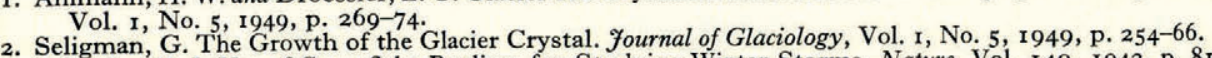

2. Seligman, G. The Growth of the Replicas for Studying Winter Storms. Nature, Vol. 149, 1942, p. 81.

3. Schaefer, V. J. Use of Snowflake Replicas for Studying Winter Storms. Nature, Physical Review, Vol. 62, Nos. 9-10,

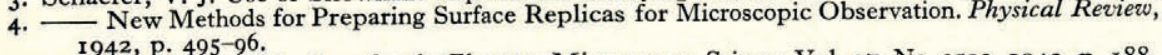

5. I942, p. 495-96. Replicas for the Electron Microscope. Science, Vol. 97, No. 2512, 1943, p. 188.

5. - Surface Replicas Containing Dye for Use in the Light Microscope. Metal Progress, Vol. 44, 1943, p. $72-75$.

\section{THE SPECIFIC GRAVITY OF ICE}

Professor P. L. Mercanton has sent the writer the results of some measurements of the density of clear ice at $0^{\circ} \mathrm{C}$. in the Glacier de Saleinaz in r917. These show densities of $0.9080,0.9013,0.9105$ and 0.9050 respectively, giving a mean of $0.9059 \mathrm{gm}$./cc. Taken in conjunction with several measurements of ice with air bubbles, yielding values below 0.90 , these results lead Professor Mercanton to the conclusion that for practical purposes it is preferable to use 0.90 rather than the usual $0.9 \mathrm{I}$ as the density of glacier ice.

It seemed hard to account for glacier ice if really clear, that is to say bubble-free, having a different specific gravity to other ice. The problem is also complicated by the fact that the salt solutions now proved to be present in glacier ice would tend to increase rather than decrease its density. The writer therefore submitted Professor Mercanton's note to Professor K. Lonsdale who replied:

"The theoretical density for pure ice derived from accurate measurements of the lattice constant of ice formed from distilled water at $0^{\circ} \mathrm{C}$. is $0.9168 \mathrm{gm} . / \mathrm{cc}$. Any deviation from this value must signify

(i) the presence of impurity, including possibly air,

(ii) a variation of lattice constant, or

(iii) a mosaic structure for the ice crystals in which there are gaps between the boundaries larger than normal molecular distances, though perhaps not large enough to allow inclusions.

Professor Lonsdale added that in her opinion (ii) would be impossible unless impurities were present which had not remained between the glacier crystals of the ice mosaic but had entered the lattice. In so far as (iii) is concerned Professor Lonsdale thought it possible that foreign atoms or groups of atoms might go between the individual crystals of the mosaic in such a way as to give large gaps, the ice crystals being held apart by sparsely distributed impurity atoms, and in that case making a reduction on balance greater than the added weight of the impurity.

The writer's view is that Professor Mercanton's generalization of 0.90 is a convenient one to adopt, especially as entirely bubble-free ice is not common. The true structure and composition of clear glacier ice await their final determination.

G. Seligman 